\title{
Effect of music during exercise on rate of perceived exertion \& mood status
}

\author{
Kumar $\mathbf{K}^{1}$, Pavithran $\mathbf{P}^{2}$ Das S.K $\mathbf{K}^{3}$ \\ ${ }^{1}$ Dr Kiran Kumar, Postgraduate in Physiology, ${ }^{2}$ Dr Purushothaman Pavithran, Assistant Professor of Physiology, \\ ${ }^{3}$ Dr Shirahatti Krishna Das, Professor \& Head of Physiology; all authors are affiliated with A J Institute of Medical \\ Sciences and Research, Centre, Kuntikana, Mangalore, Karnataka, India.
}

Address for Correspondence: Dr. Purushothaman Pavithran, Assistant Professor of Physiology, A J Institute of Medical Sciences and Research Centre, Mangalore, Karnataka, India. Email: drpavithran@outlook.com

\begin{abstract}
Background: Music has been used in boosting performance during various exercise and sports events. This present study was planned to assess the effect of music on mood status and rate of perceived exertion during jumping rope task. Methods: The study included sixty healthy students (males 30, female 30) with a mean age of $18 \pm 2$ years. Subjects were randomly assigned into three different groups $(n=20$ in each). Group 1 fast music (120bpm), group 2 slow music (90bpm) and group 3 no music group. Subjects jumped rope while listening to music for fifteen minutes or until they reported fatigue, whichever was earliest. Blood pressure, respiratory rate, rate pressure product and Profile of Mood Status was recorded before and after exercise. Results: There was a significant increase in cardiorespiratory parameters, in group $1(\mathrm{P}<0.05)$ compared to Group 2 and Group 3. Results, also revealed significant decrease in fatigue and negative mood dimensions $(\mathrm{P}<0.001$ for both) in group 1. Rate of Perceived exertion assessed after completing the exercise task was significantly lower in the group 2 compared to other two groups. Conclusion: It is concluded that exercising combined with upbeat fast music may have a positive effect on various mood parameters and cardiovascular parameters during exercise, but the same need not be true with regard of rate of perceived exertion.
\end{abstract}

Keywords: Self-selected music, Rate of perceived exertion, Mood status, exercise, Students

\section{Introduction}

Music, like language has been associated with every culture and every known civilization [1]. The use of music in daily life has been reported in scripts from ancient Greek, China, India and Rome. It has also been known to have therapeutic properties since ages. Music has been used to treat soldiers in pain and traumatic injuries during World War I and II [2]. It has also been found to reduce sleeping problems [3], to improve speed of spatial processing and the accuracy of linguistic processing [4], has been used as an ergogenic aid during various physical activities and sports [5] and has also been reported to hasten post exercise recovery [6], decrease rate of perceived exertion (RPE) and also lower cardiovascular response to exercise [7]. There is a long history of beneficial effects of using music in

Manuscript received $10^{\text {th }}$ August 2016

Reviewed: $24^{\text {th }}$ August 2016

Author Corrected: $5^{\text {th }}$ September 2016

Accepted for Publication $20^{\text {th }}$ September 2016 sports and exercise. The ability of music to increase physical work activity has been documented for over 2800 years from ancient Greece, where the kithara, a harp like instrument and flute was played during the Olympic games with the goal of improving performance during sporting activities [8].

Mood has been identified to be a central element of human behavior [9]. It is defined as the as a feeling, state or prolonged emotion that influences the whole of one's psychic life, which can affect perceived health, personal confidence, one's perceptions of the world around [10].

Music, a well-established source of entertainment is also known to have an influence on mood $[11,12]$. It has been reported to capture attention, lift spirit, generate emotion, change or regulate mood, increase work 
output, reduce inhibition and encourage rhythmic movements- all of which have potential applications in sports and exercise [13]. Exercise also has been reported to improve mood status [14]. Exercise has been suggested as an adjunct intervention in patients with mental disorders as it has been reported to reduce anxiety and depression [15].

It is a well-known fact that individuals have a tendency to monitor their mood status and also try to self-regulate their mood status to comfortable levels [9]. The authors have reported the use of music and exercise as tools to regulate mood status. Mood and emotion also plays an important role in sports performance. Music as a tool to dispel bad mood in sports persons has also been reported [16]. Studies have reported conflicting reports of music in its effect on mood status during sporting activities. No significant change in mood status was reported by a study on music intervention on emotional state in runners [17].

This study also highlighted the importance of music selection to play a role in improving emotional states during physical activity. In the present study we evaluated the combined effect of self-selected music and exercise on mood regulation. Not many studies from India have reported the acute effects of selfselected fast music in student's emotional status during physical exertion.

\section{Methodology}

The present study was carried out in the department of Physiology, A J Institute of Medical Science and Research Centre, Mangalore, Karnataka, India. We recruited 60 undergraduate medical students (mean age $18 \pm 2$ years) of both gender (Thirty males, thirty females). Participants were selected through simple random sampling technique. The research and ethics committees of our institute approved the study protocol.
Written informed consent was also obtained from each participant prior to the study.

Students were randomly assigned to three different groups namely group 1- fast music, group 2- slow music and group 3 no music (Controls). The study protocol involved each doing a jumping rope task for fifteen minutes in light comfortable clothing. Subjects jumped rope while listening to the selected music. The fast music (tempo>120 bpm) used in the present study was self-selected by the participants in group 1, whereas the music with slow tempo was selected by the researcher $(<90 \mathrm{bpm})$. These conditions were meant to ensure that there was a considerable difference between the beat and tempo of each selection of music that was played throughout the study. The music was played using Samsung galaxy tab 2 through an Iball 2.1 speaker system.

Height and weight was measured and BMI calculated. Subjects respiratory rate and blood pressure was recorded before and after the skipping task. The participants were asked to exercise for 15 minutes or till they reported fatigue, whichever was earliest.

The subjects were asked to complete the Profile of Mood status (POMS) and Borg's Rate of Perceived Exertion scale (RPE) before and after the exercise [18, 19]. Throughout the study it was made sure that the World Medical Association Declaration of Helsinki regarding ethical conduct of research involving human subject was complied with.

Statistical Analysis: Data obtained are expressed as mean \pm SD unless otherwise mentioned. Comparison of parameters was done by using one-way ANOVA and T Test. $\mathrm{P} \leq 0.05$ was considered as significant. SPSS software version-22 for windows based PC was used for statistical analysis.

\section{Results}

Baseline data for all the three groups is given in table 1. Table 2 shows the blood pressure, respiratory rate (RR) and rate pressure product (RPP) compared among the three group at baseline and after exercise. Systolic pressure (SP), Diastolic pressure (DP), RR and RPP were comparable at baseline. Whereas after exercise, SP, DP, RR and RPP were significantly different across groups. Within group analysis (Table 3 ) showed significant changes in SP, RR and RPP $(\mathrm{P}=0.0001$ for all), with the exception of DP which did not show any significant change following exercise in all three groups.

Table 4 shows the mood status parameters compared at baseline and after exercise. Anger, confusion, depression and tension parameters were significantly different across the three groups at baseline and after exercise. No significant change in fatigue $(\mathrm{P}=0.056)$ and vigor $(\mathrm{P}=0.596)$ was observed at baseline across the three groups. Following exercise significant difference was observed in fatigue $(\mathrm{P}=0.004)$ and vigor $(\mathrm{P}=0.0001)$. 
Table-1: Baseline parameters of all the three groups. Data expressed as mean \pm SD

\begin{tabular}{|c|c|c|c|c|}
\hline & $\begin{array}{c}\text { Fast music } \\
(\mathbf{n = 2 0})\end{array}$ & $\begin{array}{c}\text { Slow music } \\
(\mathbf{n = 2 0})\end{array}$ & $\begin{array}{c}\text { No music } \\
(\mathbf{n = 2 0})\end{array}$ & P value \\
\hline Age (years) & $18.2 \pm 0.41$ & $18.1 \pm 0.30$ & $18.2 \pm 0.41$ & 0.631 \\
\hline Height (mts) & $1.63 \pm 0.08$ & $1.56 \pm 0.08$ & $1.52 \pm 0.07$ & 0.494 \\
\hline Weight $(\mathrm{Kgs})$ & $53.4 \pm 6.01$ & $55.45 \pm 9.84$ & $57.1 \pm 12.4$ & 0.002 \\
\hline BMI $\left(\mathrm{Kg} / \mathrm{m}^{2}\right)$ & $20.06 \pm 1.7$ & $22.85 \pm 4.46$ & $24.19 \pm 3.77$ & \\
\hline
\end{tabular}

BMI: body mass index.

Table-2: Blood pressure, respiratory rate and rate pressure product in all three groups compared before and after exercise. Data expressed as mean \pm SD.

SP: systolic pressure, DP: Diastolic Pressure, RR: respiratory rate; RPP: rate pressure product.

\begin{tabular}{|c|c|c|c|c|c|c|c|c|}
\hline & $\begin{array}{c}\text { Fast } \\
\text { Music }\end{array}$ & $\begin{array}{c}\text { Slow } \\
\text { Music }\end{array}$ & $\begin{array}{c}\text { No } \\
\text { Music }\end{array}$ & $\begin{array}{c}P \\
\text { value }\end{array}$ & $\begin{array}{c}\text { Fast } \\
\text { Music }\end{array}$ & $\begin{array}{l}\text { Slow } \\
\text { Music }\end{array}$ & No Music & P value \\
\hline & \multicolumn{3}{|c|}{ Before exercise } & & \multicolumn{3}{|c|}{ After exercise } & \\
\hline $\mathrm{SP}(\mathrm{mm} \mathrm{Hg})$ & $111 \pm 12$ & $114 \pm 7$ & $116 \pm 11$ & 0.148 & $150 \pm 12$ & $129 \pm 10$ & $150 \pm 11$ & 0.001 \\
\hline $\mathrm{DP}(\mathrm{mm} \mathrm{Hg})$ & $73 \pm 9$ & $71 \pm 11$ & $75 \pm 10$ & 0.497 & $77 \pm 6$ & $67 \pm 6$ & $80 \pm 6$ & 0.001 \\
\hline RR(breaths/min) & $13 \pm 1$ & $14 \pm 2$ & $14 \pm 2$ & 0.673 & $30 \pm 5$ & $23 \pm 3$ & $26 \pm 5$ & 0.0001 \\
\hline RPP & $95 \pm 12$ & $96 \pm 10$ & $95 \pm 15$ & 0.951 & $192 \pm 28$ & $154 \pm 17$ & $181 \pm 22$ & 0.0001 \\
\hline
\end{tabular}

Table-3: Blood pressure, respiratory rate and rate pressure product in all three groups compared by paired $t$ test. Data expressed as mean \pm SD.

\begin{tabular}{|c|c|c|c|c|c|c|c|c|c|}
\hline & \multicolumn{2}{|c|}{ Fast Music } & \multirow{2}{*}{$\begin{array}{c}P \\
\text { value }\end{array}$} & \multicolumn{2}{|c|}{ Slow Music } & \multirow{2}{*}{$\begin{array}{c}P \\
\text { value }\end{array}$} & \multicolumn{2}{|c|}{ No Music } & \multirow{2}{*}{$\begin{array}{c}\mathbf{P} \\
\text { value }\end{array}$} \\
\hline & Before & After & & Before & After & & Before & After & \\
\hline $\mathrm{SP}(\mathrm{mm} \mathrm{Hg})$ & $111 \pm 12$ & $150 \pm 12$ & 0.0001 & $114 \pm 7$ & $129 \pm 10$ & 0.0001 & $116 \pm 11$ & $150 \pm 11$ & 0.0001 \\
\hline $\mathrm{DP}(\mathrm{mm} \mathrm{Hg})$ & $73 \pm 9$ & $77 \pm 6$ & 0.169 & $71 \pm 11$ & $67 \pm 6$ & 0.130 & $75 \pm 10$ & $80 \pm 6$ & 0.085 \\
\hline RR(breaths/min) & $13 \pm 1$ & $30 \pm 5$ & 0.0001 & $14 \pm 2$ & $23 \pm 3$ & 0.0001 & $14 \pm 2$ & $26 \pm 5$ & 0.0001 \\
\hline RPP & $95 \pm 12$ & $192 \pm 28$ & 0.0001 & $96 \pm 10$ & $154 \pm 17$ & 0.0001 & $95 \pm 15$ & $181 \pm 22$ & 0.0001 \\
\hline
\end{tabular}

SP: systolic pressure, DP: diastolic Pressure, RR: respiratory rate; RPP: rate pressure product 
Table-4: Mood status parameters in all three groups before and after exercise. Data expressed as mean \pm SD

\begin{tabular}{|c|c|c|c|c|c|c|c|c|}
\hline & Fast Music & $\begin{array}{l}\text { Slow } \\
\text { Music }\end{array}$ & No Music & $P$ value & $\begin{array}{c}\text { Fast } \\
\text { Music }\end{array}$ & $\begin{array}{c}\text { Slow } \\
\text { Music }\end{array}$ & No Music & $P$ value \\
\hline & \multicolumn{3}{|c|}{ Before exercise } & & \multicolumn{3}{|c|}{ After exercise } & \\
\hline Anger & $8 \pm 3.07$ & $5.25 \pm 2.53$ & $4.6 \pm 2.4$ & 0.0001 & $1.3 \pm 1.2$ & $5.05 \pm 2.8$ & $4.95 \pm 2.92$ & 0.0001 \\
\hline Confusion & $8.15 \pm 1.69$ & $5.75 \pm 1.99$ & $6.05 \pm 1.70$ & 0.0001 & $4.65 \pm 1.13$ & $5.65 \pm 1.49$ & $6.47 \pm 1.87$ & 0.003 \\
\hline Depression & $10.65 \pm 3.93$ & $6.45 \pm 2.81$ & $6.45 \pm 3.36$ & 0.0001 & $1.90 \pm 1.77$ & $7.2 \pm 3.66$ & $6.91 \pm 3.65$ & 0.0001 \\
\hline Fatigue & $4.65 \pm 2.49$ & $3.5 \pm 1.8$ & $3.1 \pm 1.7$ & 0.056 & $1.9 \pm 1.2$ & $3.8 \pm 2.3$ & $3.9 \pm 2.3$ & 0.004 \\
\hline Tension & $10.05 \pm 1.87$ & $6.95 \pm 1.66$ & $6.9 \pm 1.91$ & 0.0001 & $3.35 \pm 1.56$ & $7.2 \pm 1.61$ & $7.05 \pm 2.43$ & 0.0001 \\
\hline Vigor & $6.6 \pm 2.3$ & $5.7 \pm 3.0$ & $6.25 \pm 2.93$ & 0.596 & $9.7 \pm 2.3$ & $3.95 \pm 1.76$ & $6.5 \pm 2.66$ & 0.0001 \\
\hline
\end{tabular}

Table-5: Mood status parameters in all three groups before and after exercise. Data expressed as mean \pm SD

\begin{tabular}{|c|c|c|c|c|c|c|c|c|c|}
\hline & \multicolumn{2}{|c|}{ Fast Music } & \multirow{2}{*}{ P value } & \multicolumn{2}{|c|}{ Slow Music } & P value & \multicolumn{2}{|c|}{ No Music } & P value \\
\cline { 7 - 10 } & Before & After & & Before & After & & Before & After & \\
\hline Anger & $8 \pm 3$ & $1.3 \pm 1.2$ & 0.0001 & $5.3 \pm 2.5$ & $5 \pm 2.8$ & 0.75 & $4.6 \pm 2.4$ & $4.95 \pm 2.9$ & 0.79 \\
\hline Confusion & $8 \pm 1.6$ & $4.6 \pm 1.1$ & 0.0001 & $5.6 \pm 1.9$ & $5.5 \pm 2.8$ & 0.62 & $6.05 \pm 1.7$ & $6.4 \pm 1.8$ & 0.30 \\
\hline Depression & $10.65 \pm 3.9$ & $1.9 \pm 1.77$ & 0.0001 & $6.5 \pm 2.8$ & $7.2 \pm 3.6$ & 0.07 & $6.45 \pm 3.3$ & $6.9 \pm 3.6$ & 0.34 \\
\hline Fatigue & $4.65 \pm 2.5$ & $1.9 \pm 1.20$ & 0.01 & $3.5 \pm 1.8$ & $3.8 \pm 2.3$ & 0.22 & $3.1 \pm 1.7$ & $3.9 \pm 2.3$ & 0.02 \\
\hline Tension & $10.0 \pm 1.8$ & $3.35 \pm 1.5$ & 0.001 & $6.9 \pm 1.6$ & $7.2 \pm 1.6$ & 0.21 & $6.9 \pm 1.9$ & $7 \pm 2.4$ & 0.79 \\
\hline Vigor & $6.6 \pm 2.3$ & $9.7 \pm 2.3$ & 0.0001 & $5.7 \pm 3$ & $3.9 \pm 1.7$ & 0.05 & $6.2 \pm 2.9$ & $6.6 \pm 2.6$ & 0.60 \\
\hline POMS & $35 \pm 10$ & $3.54 \pm 4.6$ & 0.0001 & $22 \pm 8$ & $25 \pm 9$ & 0.02 & $21 \pm 8$ & $23 \pm 10$ & 0.29 \\
\hline
\end{tabular}

Within group comparison of various mood parameters (Table 5) demonstrated very significant changes in group I. Anger, confusion and depression was found to be significantly reduced in group $\mathrm{I}(\mathrm{P}=0.0001$ for all). Fatigue $(\mathrm{P}=0.01)$ and Tension $(\mathrm{P}=0.001)$ also showed significant reduction following exercise in group I. Vigor was observed to be significantly higher following exercise in this group $(\mathrm{P}=0.001)$. The overall effect was the significant lowering of POMS following exercise $(\mathrm{P}=0.0001)$ in the group $\mathrm{I}$.

In Group II a significant increase in depression $(\mathrm{P}=0.07)$ and a decrease in vigor $(\mathrm{P}=0.05)$ was observed following exercise. None of the other parameters showed any significant change in this group. POMS $(\mathrm{P}=0.02)$ showed a slight increment as compared to baseline.

In group III, after exercise except for fatigue there was no significant change in any of the mood parameters including POMS. Fatigue showed a significant increase in this group following exercise $(P=0.02)$. Statistically significant difference was observed in RPE between group I and group II; group II and group III (P = 0001 for both) (fig 1). RPE was lowest in group II as compared to the other two groups following exercise.

\section{Discussion}

Music as an adjuvant to various ongoing therapy has been reported very extensively. The basis for this use of music, is its ability to reduce anxiety and pain in patients [2]. It has also been used in motivating athletes involved in events like swimming [20], treadmill walking $[21,22]$ aerobic exercise wherein music has been reported to enhance endurance and exercise tolerance. The present study was carried out to compare the effect of self-selected fast music with that of slow music and no-music music during exercise and its effects on mood status and rate of perceived exertion. In the present study we found self-selected fast music to 
improve vigor, reduce fatigue and also to decrease anger, depression, confusion and tension. This is in line with the reports wherein subjects exposed to selfselected music has been reported to be more efficient, to perceive lowest distraction, highest enjoyment, liking and appropriateness, and to experience a reduction in tension-anxiety [23]. Studies have also reported the positive effect of fast music, wherein the authors have reported an increase in duration of the exercise performed [24]. Japanese folk music and aerobic dance music has also been reported to reduce fatigue in women during bench stepping exercise. The same study has also reported an increase in vigor and less confusion with aerobic dance music compared to those on no music [25]. Studies have also reported that listening to fast, upbeat music during exercise may be beneficial for untrained runners but counterproductive for trained runners [26].

Perceived exertion is defined as the subjective intensity of effort, strain discomfort and/or the fatigue that is experienced during an exercise [27]. RPE as assessed by Borgs [19] 15-point scale in our study highlighted the fact the subjects on fast music had the highest RPE compared to those on slow music, but not no-music. This is in contrast to most of the studies on fast music and RPE, that having reported a lowering of RPE with fast music compared to slow music. In line with our study findings, a study on the effects of different types of music on HR, RPE, and time to exhaustion during treadmill work have reported slow music to lower RPE [22]. A study assessing the effect of music listening during exercise have highlighted its positive effect on exercise and RPE, but have not mentioned the tempo of the music used in that study [28]. In contrast to our study findings are findings of the study which has reported fast music to lower RPE levels during 20 minutes of moderate intensity exercise.

The authors of the same study have also reported slow music to also lower RPE. The second part of their findings are in line with our study findings [29]. There are studies which have reported no effect of fast music on RPE [30, 31]. In the present study as the subjects were involved in exercise, SP, RR and RPP product which is an index of myocardial oxygen consumption [32] showed a significant increase following exercise. In line with the present study finding is the report on the effect of fast and slow music on cardiovascular and hemodynamic parameters in steady state exercise wherein an increase in oxygen consumption, cardiac output and minute ventilation and a decrease in systemic vascular resistance was reported [33]. In contrast to our study findings, no significant difference in pulse rate and bicycle ergometer riding time was reported while using fast music, slow and no music [34]. In the present study, even though there was significant increase in SP, RR and RPP in the fast music group as compared to the slow music group, there was no significant difference in these parameters between fast music and no music group. The mechanism by which music regulates stress arousal, and emotions has been proposed to be by way of activating brainstem responses. Music has been reported to modulate heart rate, pulse, blood pressure, body temperature, skin conductance, and muscle tension [35]. Tempo is also said to play an important role in this mechanism, wherein slow music is said to decrease HR, RR and BP, whereas fast music has the opposite effect [36]. Music has also been reported to increase cerebral blood flow in areas of the brain concerned with reward and reinforcement mechanism [35]. This could be a one of the mechanism involved in the increased performance and other positive effects of observed in subjects listening to fast music in the present study group.

Most of the studies on the effect of fast music, slow music and no music have reported self-selected fast music to be better in terms of mood status and rate of perceived exertion during exercise task. From the present study we conclude that self-selected fast music compared to slow and no music is better tool to control mood, but the same could not be said for its role on RPE as we find our results are in contrary to this. In the present subset of subjects, we could not find selfselected fast music to lower RPE, even though it improved mood parameters in them. The study also leaves some unanswered questions as to why there was no significant difference between POMS values, cardiovascular parameters and RPE between no music and fast music.

Acknowledgment- The authors are thankful to the management of A J Institute of Medical Sciences and Research Centre for providing us the opportunity to carry out this work in the department of Physiology. We also thank the makers of Paperpile, reference manager, which has helped us immensely while preparing this manuscript.

Author contribution: All authors have read and accepted the final version of the manuscript and has approved its submission. 
Funding: Nil, Conflict of interest: None initiated, Permission from IRB: Yes

\section{References}

1. Trehub SE, Trainor LJ, Unyk AM. Music and speech processing in the first year of life. Adv Child Dev Behav 1993; 24:1-35. DOI:10.1016 /S0065-2407 (08) 60298-0.

2. Mattei TA, Rodriguez AH. Music therapy as an adjuvant therapeutic tool in medical practice: an evidence-based summary.OAEvidence-Based Medicine 2013;1(1):1-8. DOI: 10.13172/2053-2636-1-1-405.

3. Harmat L, Takács J, Bódizs R. Music improves sleep quality in students. J Adv Nurs. 2008 May;62(3):32735. DOI: 10.1111/j.1365-2648.2008.04602.x

4. Angel LA, Polzella DJ, Elvers GC. Background music and cognitive performance. Percept Mot Skills. 2010 Jun; 110 (3 Pt 2):1059-64.DOI:10.2466/pms. 110. C. $1059-1064$

5. Waterhouse J, Hudson P, Edwards B. Effects of music tempo upon submaximal cycling performance. Scand J Med Sci Sports. 2010 Aug;20(4):662-9. DOI: 10.1111/j.1600-0838.2009.00948.x

6. Savitha D, Mallikarjuna RN, Rao C. Effect of different musical tempo on post-exercise recovery in young adults. Indian J Physiol Pharmacol. 2010 Jan; 54(1):32-6. PMID: 21046917.

7. Arazi H, Asadi A, Purabed M. Physiological and Psychophysical Responses to Listening to Music during Warm-Up and Circuit-Type Resistance Exercise in Strength Trained Men. J Sports Med. Hindawi Publishing Corporation; 2015 Aug 4 [cited 2015 Oct 1]; 2015.Available from: http: // www. hindawi. com/ journals /jsm/ 2015 /389831 / DOI: 10.1155/2015/ 389831 .

8. Trappe H-J. Role of music in intensive care medicine. Int J Crit Illn Inj Sci. 2012 Jan;2(1):27-31. DOI: $10.4103 / 2229-5151.94893$.

9. Thayer RE, Newman JR, McClain TM. Selfregulation of mood: strategies for changing a bad mood, raising energy, and reducing tension. J Pers Soc Psychol. 1994 Nov;67(5):910-25. PMID: 7983582.

10. Clark AV. Psychology of Moods: New Research. Nova Science Publishers; 2006.
11. McCraty R, Barrios-Choplin B, Atkinson M, Tomasino D. The effects of different types of music on mood, tension, and mental clarity. Altern Ther Health Med. 1998 Jan;4(1):75-84. PMID: 9439023.

12. van der Zwaag MD, Janssen JH, Westerink JHDM. Directing Physiology and Mood through Music: Validation of an Affective Music Player. Affective Computing, IEEE Transactions on. 2013 Jan;4(1):5768. DOI: 10.1109/T-AFFC.2012.28.

13. Terry P.C KCI. Psychophysical effects of music in sport and exercise: an update on theory, research and application. In: Katsikitis M, editor. Psychology bridging the Tasman: Science, culture and practice Proceedings of the 2006 Joint Conference of the Australian Psychological Society and the New Zealand Psychological Society. p. 415-9.

14. Lane AM, Crone-Grant D, Lane H. Mood changes following exercise. Percept Mot Skills. 2002;94(3): 732-4. DOI: 10.2466/pms.2002.94.3.732.

15. Sharma A, Madaan V, Petty FD. Exercise for mental health. Prim Care Companion. J Clin Psychiatry. 2006; 8(2):106. PMC1470658.

16. Tiwari A. Assessment of Strategies Used to Alternation of Bad Mood by Indian Hockey Players of India. International Educational E-Journal, [Internet]. 2013; 2(3). Available from: http: //www.oiirj.org/ ejournal / july-aug-sept2013/15.pdf

17. Lane AM, Davis PA, Devonport TJ. Effects of music interventions on emotional States and running performance. J Sports Sci Med. 2011 Jun 1;10(2):4007. PMC3761862.

18. Mackenzie B. Borg Scale - Rate of perceived exertion (RPE) [Internet]. [cited 2016 Mar 9]. Available from: http://www.brianmac.co.uk/borgscale.htm\#ref

19. Borg GA. Psychophysical bases of perceived exertion. Med Sci Sports Exerc. 1982;14(5):377-81. PMID: 7154893.

20. Karageorghis CI, Hutchinson JC, Jones L, Farmer HL, Ayhan MS, Wilson RC, et al. Psychological, psychophysical, and ergogenic effects of music in swimming. Psychol Sport Exerc. 2013 Jul;14(4):560-8. DOI: 10.1016/j.psychsport.2013.01.009.

21. Karageorghis CI, Mouzourides DA, Priest D-L, Sasso TA, Morrish DJ, Walley CJ. Psychophysical and 
ergogenic effects of synchronous music during treadmill walking. J Sport Exerc Psychol. 2009 Feb; 31(1):18-36. DOI: 10.1123/jsep.31.1.18.

22. Copeland BL, Franks BD. Effects of types and intensities of background music on treadmill endurance. J Sports Med Phys Fitness. 1991 Mar;31(1):100-3. PMID: 1861474.

23. Cassidy G, Macdonald R. The effects of music choice on task performance: A study of the impact of self-selected and experimenter-selected music on driving game performance and experience. Musicae Scientiae. 2009; 13(2): 357-86. DOI: 10.1177/ 102986490901300207.

24. Thakur AM, Yardi SS. Effect of different types of music on exercise performance in normal individuals. Indian J Physiol Pharmacol. 2013 Oct;57(4):448-51. PMID: 24968586.

25. Hayakawa Y, Miki H, Takada K, Tanaka K. Effects of music on mood during bench stepping exercise. Percept Mot Skills. 2000 Feb;90(1):307-14. DOI: 10.2466/pms.2000.90.1.307

26. Brownley KA, McMurray RG, Hackney AC. Effects of music on physiological and affective responses to graded treadmill exercise in trained and untrained runners. Int J Psychophysiol. 1995 Apr; 19 (3) : 193-201. DOI: DOI: 10. 1016/0167-8760 (95) 00007-F.

27. Noble B, Robertson, editors. Perceived exertion. Human Kinetics. IL: Champaign; 1996.

28. Mohammadzadeh H, Tartibiyan B, Ahmadi A. The effects of music on the perceived exertion rate and performance of trained and untrained individuals during progressive exercise. Facta Universitatis-Series: Physical Education and Sport. 2008;6(1):67-74.
29. Potteiger JA, Schroeder JM, Goff KL. Influence of music on ratings of perceived exertion during 20 minutes of moderate intensity exercise. Percept Mot Skills. 2000 Dec;91(3 Pt 1):848-54. DOI: 10.2466/pms. 2000.91.3.848.

30. Schwartz SE, Fernhall B, Plowman SA. Effects of Music on Exercise Performance. J Cardiopulm Rehabil Prev. journals. lww.com; 1990 Sep; 10(9): 312. PMC3944555.

31. Jarraya M, Chtourou H, Aloui A, Hammouda O, Chamari K, Chaouachi A, et al. The Effects of Music on High-intensity Short-term Exercise in Well Trained Athletes. Asian J Sports Med. 2012 Dec;3(4):233-8. PMC3525819.

32. Gobel FL, Norstrom LA, Nelson RR, Jorgensen CR, Wang Y. The rate-pressure product as an index of myocardial oxygen consumption during exercise in patients with angina pectoris. Circulation. 1978 Mar; 57(3):549-56. DOI:10.1161/01.CIR.57.3.549.

33. Birnbaum L, Boone T, Huschle B. Cardiovascular responses to music tempo during steady-state exercise. $\mathbf{J}$ Exerc Physiol Online. 2009;12(1):50-7.

34. Coutts CA. Effects of Music on Pulse Rates and Work Output of Short Duration. Research Quarterly American Association for Health, Physical Education and Recreation. 1965; 36(1): 17-21. DOI: 10.1080/ 10671188. 1965.10614652.

35. Chanda ML, Levitin DJ. The neurochemistry of music. Trends Cogn Sci. 2013 Apr;17(4):179-93. DOI: 10.1016/j.tics.2013.02.007.

36. Bernardi L, Porta C, Casucci G, Balsamo R, Bernardi NF, Fogari R, et al. Dynamic interactions between musical, cardiovascular, and cerebral rhythms in humans. Circulation. 2009 Jun 30;119(25):3171-80. DOI:10.1161/CIRCULATIONAHA.108.806174.

\section{How to cite this article?}

Kumar K, Pavithran P Das S.K. Effect of music during exercise on rate of perceived exertion \& mood status. Int J Med Res Rev 2016;4(9):1706-1712.doi:10.17511/ijmrr. 2016.i09.32. 